\title{
ESTIMATION OF THE PORES AGGLOMERATION EFFECT ON THE COMPRESSIVE BEHAVIOR OF METALLIC FOAMS: INFILTRATION AND POWDER METALLURGY STUDY CASES
}

\author{
Juan C. CARranza ${ }^{1}$, Berhta Y. CAsas ${ }^{1}$, Ismeli Alfonso ${ }^{1 *}$, \\ Luis PÉrEZ ${ }^{2}$, Robin A.L. DrEW ${ }^{3}$, JORGE A. VERdUZCO ${ }^{4}$, \\ IGNACIO A. FIGUEROA ${ }^{5}$ \\ ${ }^{1}$ Instituto de Investigaciones en Materiales, Unidad Morelia, \\ Universidad Nacional Autónoma de México, Campus Morelia UNAM, \\ Antigua Carretera a Pátzcuaro No. 8701, Col. Ex-Hacienda de San José de la \\ Huerta. C.P. 58190, Morelia, Michoacán, México \\ ${ }^{2}$ Department of Mechanical Engineering, Universidad Técnica Federico \\ Santa María, Av. España 1680, Casilla 110-V, Valparaíso, Chile \\ ${ }^{3}$ Department of Mechanical, Industrial and Aerospace Engineering, \\ Concordia University, 1455 Maisonneuve Blvd West, Montreal, QC, \\ Canada $H 3 G 1 M 8$ \\ ${ }^{4}$ Instituto de Investigacin en Metalurgia y Materiales, Universidad \\ Michoacana de San Nicolás de Hidalgo. Edificio U, Ciudad Universitaria, \\ C.P. 58190, Morelia, Michoacán, México \\ ${ }^{5}$ Instituto de Investigaciones en Materiales, Universidad Nacional Autónoma \\ de México, Circuito Interior SN. Ciudad Universitaria, Del. Coyoacán, \\ C.P. 58190, Ciudad de México, México
}

[Received: 14 June 2019. Accepted: 30 April 2020]

doi: 10.7546/JTAM.50.20.02.07

\begin{abstract}
The present work investigates the effect of the pores arrangement on the elastic compressive behavior of metallic foams. Models with porosities ranging from 45 to $65 \%$ were obtained using Finite Element Analysis (FEA), with two arrangements: agglomeration similar to that obtained in the powder metallurgy process (PM), and a distribution without agglomeration obtained using infiltration. Experimental foams were obtained for validating purposes. Results show that for both PM and infiltration cases the estimated Young's moduli (E) decrease with the increase in porosity. Moreover, the degree of agglomeration $(m)$ was introduced as a measure of the pore distribution, which presented an important effect on $\mathrm{E}$. The magnitude of $\mathrm{E}$, which was obtained by means of numerical simulation of uniaxial compression, was significantly lower (close to 70\%) for agglomerated pores (high $m$ values) than that for the non-agglomerated condition. Besides, Young's moduli obtained by simulating the foams manufactured by PM and infiltration were in excellent agreement with the experimentally manufactured foam values. These results remark the
\end{abstract}

\footnotetext{
${ }^{*}$ Corresponding author e-mail: ialfonso@unam.mx
} 
importance of using models according to the real topology defined by the manufacturing process.

KEY WORDS: foam, FEA, DEM, agglomeration, infiltration, powder metallurgy.

\section{INTRODUCTION}

Metallic foams are a new class of materials with properties that make them excellent replacements for conventional materials in specialized applications. Their porous structure leads to a combination of low density and excellent mechanical, thermal, acoustic, and chemical properties. Literature shows that among the most common applications of these materials are energy absorbers, certain automotive parts, biomedical applications, and heat exchangers [1-4]. Foams can be divided into open and closed cell materials, and their properties highly depend on the kind of cell, among other characteristics. In the case of open cell foams important parameters to control to obtain a wide range of properties are: a) pore interconnectivity, b) percentage porosity, c) cell wall thickness and d) pore agglomeration. Processes involving metal in solid, liquid and gaseous states have been reported for production of this kind of foam, as showed Ashby et al. [1] and Banhart [2]. Among these processes are those involving the incorporation of removable space holder particles (SHPs), including sintering [5] and infiltration [6]. Typical SHPs are $\mathrm{NaCl}$ or $\mathrm{KBr}$, which can be removed by post-processing dissolution, as is shown in literature [7,8]. Perez et al. [9] demonstrated that pore networks change depending on the SHPs size, shape and arrangement prior to the final manufacturing process. The accommodation of these particles depends on the mixing process, as in the case of powder metallurgy (PM), where metal powder is mixed with the SHPs for sintering during a second step [7]; or their self-accommodation, as in the case of alloy infiltration reported by OsorioHernandez et al. [10], where a SHP preform is used into which the molten metal is infiltrated during a second step. The arrangements are different for the foams obtained using these two methods. In the case of PM it is common to obtain pore agglomeration resulting from the different sizes and densities of SHPs and metal powders, while SHPs used as preforms in the infiltration process are homogeneously arranged and distributed.

Although the analysis of both elastic and plastic regions are important, there are numerous works in literature focused only on Young's modulus (E), comparing the estimations with the results obtained from other models [11-13]. Some applications require the study of $\mathrm{E}$, such as the use of foams as biomaterials. Hence it is essential to predict $\mathrm{E}$ according to the nature of SHPs and the manufacturing process. As the validity of the estimations mostly depends on the proximity of the model 
to the real foam topology, it is fundamental to recreate closely the arrangement of the SHPs after the mixing process. Due to its modeling capability, Finite Element Analysis (FEA) is one of the methods being used to predict foams properties. It is capable of modeling different arrangements of SHPs and to simulate their effect on mechanical properties, as show different works in literature [9,11-13]. Besides FEA method, different models used for Young's modulus estimation only takes into account the density or porosity of the foams, and generally over-predict the foam strength. Among them are models with repetitive unit cell to provide the homogenized behavior of foams, including two dimensional foam/honeycomb models and three-dimensional cubic, tetrahedral, and tetrakaidecahedral models. Examples are the models of Zhu et al. [14]; Warren and Kraynik [15]; and Gan et al. [16]. In theory, are only applicable to foams with regular and periodic cells, whilst real foams are typically aperiodic, non-uniform and disordered, as show the works of Hasan [17] and Cadena et al. [18], so aspects such as agglomeration and/or cell wall thickness should also be included. Therefore, it is very important in the initial stage of modeling to generate randomly distributed spheres and this can be achieved via Computer Aided Design (CAD). Although the SHPs can be present in several shapes, the subsequent pores can be modeled as spheres in order to make the modeling process easier and deliver good estimations. Spheres minimize the model complexity and the computer requirements compared to the use of prisms. Angular pores are under higher stresses than spherical pores, for which stress is quite uniform. Nevertheless, FEA studies using rounded or angular shapes have demonstrated that the differences in the resulting Young's moduli are not significant, being then possible approximations to simple shapes without sacrificing the accuracy of the model. The number of spheres inserted in the CAD model, their sizes, and the distance between their centers control the percentage porosity, agglomeration, and interconnectivity, being essential for obtaining foam close to real topologies. Once these coordinates are generated, the porosity is produced in a second stage, by deleting the sphere volume from the container, a process executed using FEA, previously presented by Pérez et al. [19]. In these studies, Discrete Element Method (DEM) was used in order to generate these randomly distributed coordinates. This numerical method, developed by Cundall and Strack [20], is a useful tool for modeling the behavior of granular and particulate systems. It may be used for generating the final position of the SHPs in the mixing process of the metallic powder-space holder mixture used in the manufacturing process of metallic foams; or in the mixing process of SHPs used as a preform in infiltration. The distance between the centers of the resulting spheres and their interactions could be modified to create these different conditions. Besides, the use of DEM provides several advantages, one being the reduction in required computing power. As the DEM-FEA combination has demonstrated to be very useful to replicate the arrange- 
ment of pores in foams, resulting in models closer to real foams topologies, present work aims to generate randomly arranged porous networks using DEM-FEA, by attempting to reproduce the different degrees of agglomeration, obtained in PM and infiltration processes. The use of numerical modelling and simulations for predicting the elastic properties of foams could help in their design and manufacturing process selection.

\section{Modeling ANd Simulation}

In this section the technique is shown for generating two different models of foams, each one with different pore arrangements. The first tries to represent the agglomeration of the pores as the typical arrangement obtained by conventional PM incorporating removable SHPs. The second embodies the arrangement of the SHPs in the preform used in the infiltration process, where all particles touch in a random arrangement with a low quantity of pore-free space.

\subsection{COORDINATES GENERATION}

In order to generate the coordinates that best represent the two above-mentioned models, the interaction of spheres incorporated in $20 \mathrm{~mm}$ diameter and $16 \mathrm{~mm}$ high cylinders were modeled using LIGGGHTS $® D E M$ simulation software. Creating different conditions modified the level of interaction between spheres. In the case of PM the diameter of immersed spheres is significantly lower than the final diameter used in the next stage of CAD model creation, which allows pores agglomeration due to the high interconnection. On the other hand, for the infiltration case above mentioned, diameter of immersed spheres is slightly smaller than the final diameter. Therefore, interconnection is low as occurs for infiltration process. It is also important to remark that for both cases interconnectivity is present, which in reality, is obtained during incipient melting of the particles. Coordinates for modelling foams with porosities of $45 \%, 55 \%$, and $65 \%$ were generated. Three random distributions for each condition were generated in order to ensure reproducible results. Fig. 1 depicts examples of

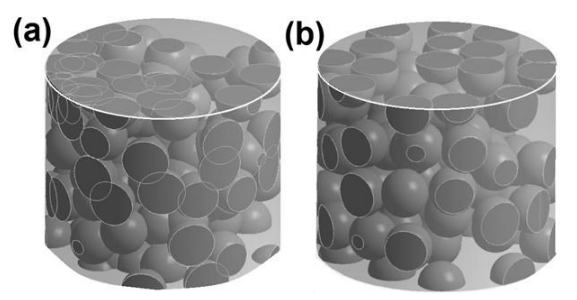

Fig. 1: Particle distributions generated using DEM for cylinders with final SHP percentages of $45 \%$, for the cases of PM (a), and infiltration (b). 
final particle distributions from the DEM generation process, showing agglomeration (Fig. 1a) typical of PM; and non-agglomeration (Fig. 1b), in the case of infiltration. These arrangements were used in the second stage of foam modelling using a porosity level of $45 \%$.

The above-generated coordinates were post-processed for use in ANSYS 16.0 Design Modeler script and to create the CAD models of the foams. The correct percentage porosity was obtained by modifying the number of particles inserted into the cylindrical geometries.

\subsection{Finite ELEMENTS MODELS}

The FEA models consisted of cylindrical specimens of $20 \mathrm{~mm}$ diameter and $16 \mathrm{~mm}$ high, with porosities of $45 \%, 55 \%$, and $65 \%$. The pores were modeled as spheres of $4 \mathrm{~mm}$ in diameter, either agglomerated or not according to previous criteria. ANSYS 18.1 FEA was used for modeling and simulation. Fig. 2 shows the cylindrical models of the foams with different porosities engendered through DEM-FEA combination. As can be observed in Figs. $2 \mathrm{a}$ to $2 \mathrm{c}$, the pores are randomly distributed with signifi-

(a)
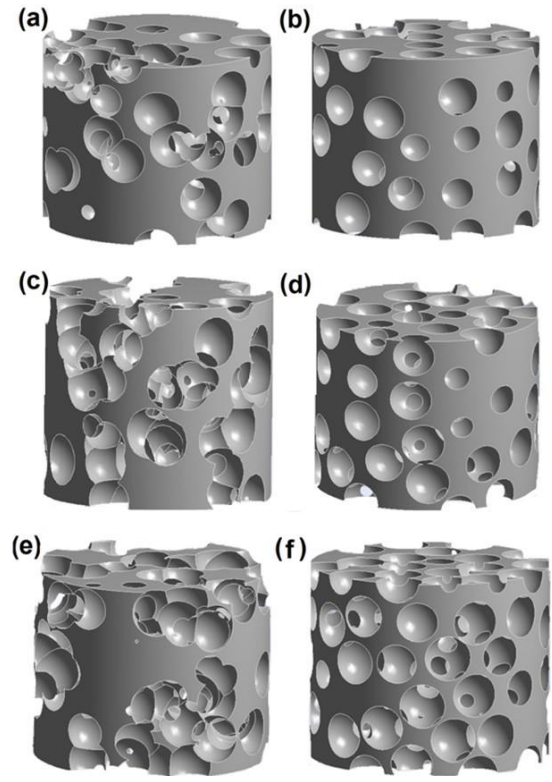

Fig. 2: Finite element models using ANSYS for foams with porosities of: (a, b) $45 \%$, (c, d) $55 \%$, and (e, f) $65 \%$. Agglomeration of pores can be observed for foams recreating PM processes (a, c and e). No agglomeration is observed for infiltration (b, d and f). 
cant agglomeration, replicating the typical arrangement obtained for PM after powder mixing. Otherwise, Figs. $2 \mathrm{~d}$ to $2 \mathrm{f}$ show foams with the same percentage of porosity as witnessed previously, but without agglomeration and observed when a preform is used in infiltration. These two case studies are representative of the final positions of the SHP after mixing during foam manufacturing. This differentiation according to the manufacturing process will allow creating models much closer to the real foam topologies, which is imperative information for improving the predictability of FEA.

\subsection{Simulations}

In order to obtain the elastic modulus, numerical experiments were performed consisting of the uniaxial compression on the nodes of the upper end of the cylindrical specimens. These stresses were determined according to the real area (area without pores) of the bottom surfaces of the cylinders in order to always apply the same stress and obtain repetitive results. Structural elements consisting of 10-node 3-D tetrahedral SOLID 187 were employed for meshing. The number of nodes ranged from 4.9 to $8.2 \times 10^{5}$, while the number of elements was from 2.5 to $4.2 \times 10^{5}$, decreasing these quantities with increasing porosity. Gradually increasing the number of elements and verifying the local stress behavior in order to ensure convergence of the numerical solution carried out mesh convergence analysis. The nodes were kept in the same plane for the upper face of the cylinder applying the coupled-node boundary condition. Young's modulus $\left(E_{z}\right)$ was obtained from the response to the compression along the $\mathrm{z}$-axis using stress $\left(s_{z}\right)$ and strain $\left(e_{z}\right)$, determined using the displacement in $z$-axis $\left(u_{z}\right)$ as follows:

$$
E_{z}=s_{z} / e_{z} .
$$

Strain was determined through Eq. 2 using a pre-established displacement to the total original height of the cylindrical specimen $\left(L_{z}\right)$ :

$$
e_{z}=u_{z} / L_{z},
$$

while the stress necessary for solving Eq. 1 is

$$
s_{z}=F_{z} / A,
$$

where $F_{z}$ is the reaction force in the $z$-axis obtained through the FEA simulation, for the nodes of the bottom end of the cylindrical specimens; while $A$ is the area of this surface. For comparative purposes Young's modulus ( $77 \mathrm{GPa}$ ) and Poisson's ratio (0.33) of Al-Si alloys reported by Joshi et al. [21] were used for the solid part of the foams. 


\section{EXPERIMENTAL}

Just for comparing and validating purposes, an $\mathrm{Al}-\mathrm{Si}-\mathrm{Cu}$ metallic foam was manufactured by infiltration. This foam was obtained introducing $\mathrm{NaCl}$ particles of $4 \mathrm{~mm}$ in diameter (as SHP preform) into a $5 \mathrm{~cm}$ diameter and $30 \mathrm{~cm}$ height AISI 314 stainless steel cylinder with a sealed base, filled to a height of $10 \mathrm{~cm}$ with the SHP in order to obtain foams of these dimensions. A $332 \mathrm{Al}-\mathrm{Si}-\mathrm{Cu}$ alloy was introduced into the cylinder over the $\mathrm{NaCl}$ preform. The cylinder was then introduced into the top hole of a Prefinsa HR-C4 electric resistance furnace, where the $\mathrm{NaCl}$ preform was infiltrated with the molten $\mathrm{Al}$ alloy by gravity when the temperature of the furnace was raised to $700^{\circ} \mathrm{C}$. The cylindrical $\mathrm{Al}-\mathrm{NaCl}$ composites formed were removed from the steel cylinder and immersed in water to dissolve the SHPs. A second foam was obtained by powder metallurgy mixing in a double cone mixer during 15 minutes Al powder with irregular shape, particle size in the range of 70-90 $\mu \mathrm{m}$ and a purity of $99.5 \%$, with $\mathrm{NaCl}$ particles ranging from $170-200 \mu \mathrm{m}$ (95\% of the particles in this range). This mixture was uniaxially compacted at $300 \mathrm{MPa}$ and sintered at $630^{\circ} \mathrm{C}$, followed by $\mathrm{NaCl}$ dissolution. Porosity percentage and cell wall thickness of the obtained foams were measured by means of the relative density and by Optical Microscopy (OM) using a Labomed Med 400 OM. Experimental Young modulus of this foam was determined averaging the linear zone of three compressive stress-strain curves, obtained for cylindrical samples of $20 \mathrm{~mm}$ in diameter and $16 \mathrm{~mm}$ in height. It was used a universal testing machine Instron 5500R at a constant crosshead speed of $0.5 \mathrm{~mm} / \mathrm{s}$. A correction was made in order to determine the zero coordinate of the linear zone applying a tangent to the point of the maximum slope of the elastic region. This correction was according to ASTM E9-09 Standard, also reported for aluminum foams by Florek et al. [22]. The compression of the Al-Si-Cu alloy without pores revealed a Young's modulus of $75 \mathrm{GPa}$, similar to the reported by Joshi et al. [21], used for simulation. This value for PM was $38.2 \mathrm{GPa}$, directly influenced by the microporosity between metallic powders originated by the compaction level.

\section{Results AND Discussion}

The graphical distribution of the displacements originated due to the applied stresses can be observed in Fig. 3 for foams with porosities of $45 \%$ (a, b) and 65\% (c,d). As can be observed, maximum displacements were obtained for the foams that replicate $\mathrm{PM}$, being $6.8 \times 10^{-11}$ and $1.43 \times 10^{-11}$ for PM and infiltration foams, respectively, and $1.87 \times 10^{-10}$ for a porosity of $45 \%$ and $8.06 \times 10^{-11}$ for a porosity of $65 \%$. This result was used for Young's modulus determination and shows that the presence of agglomeration led to a higher elastic deformation in the models.

In Fig. $4 \mathrm{a}$ it is shown the comparison of Young' moduli magnitudes for foams 

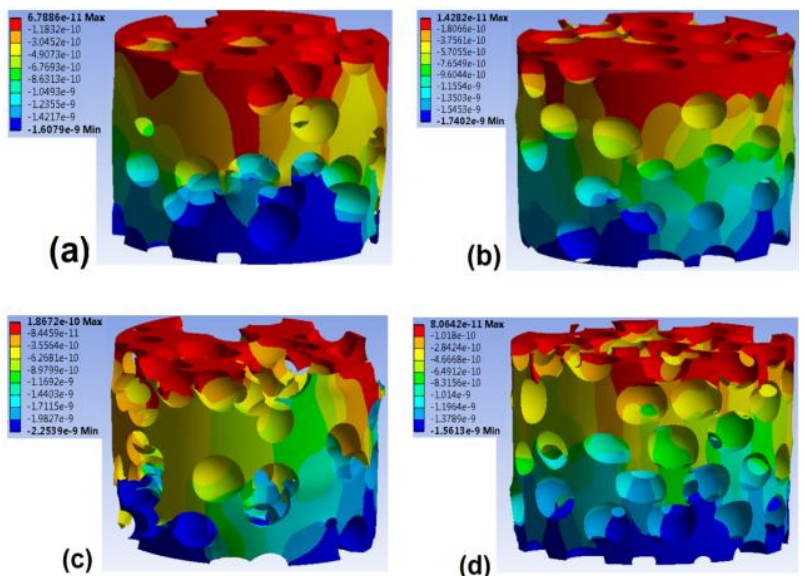

Fig. 3: Distribution of the displacements produced in the foams modeled using ANSYS 18.1, for porosities of: (a,b) $45 \%$ and (c,d) $65 \%$. Displacements were higher for foams recreating the PM process (a and c) than those for the infiltration process $(b$ and d).

with different porosities, calculated by means of numerical simulation of uniaxial compression using FEA, including conditions with agglomeration (PM) and without agglomeration (infiltration). As can be observed, the Young's modulus decreases significantly when the porosity increases in both cases. Furthermore, there are significant differences between results, notably always being higher for foams without agglomeration (infiltration). These higher values could be explained by agglomeration creating bigger pores and leading to lower strengths. In order to compare these results with literature values, the estimations obtained by models of Zhu et al. [14]; Warren and Kraynik [15]; and Gan et al. [16] were included in Fig. 4a. Their equations are as follows:

$$
\begin{array}{llrl}
\text { (Zhu et al.) } & E=\frac{1.009 E_{s} \rho^{2}}{1+1.514 \rho^{2}} ; \\
\text { (Warren and Kraynik) } & E=\frac{E_{s} \rho^{2}(11+4 \rho)}{\left(10+31 \rho+4 \rho^{2}\right)} ; \\
\text { (Gan et al.) } & E=\frac{E_{s} \rho^{2}}{1+6 \rho},
\end{array}
$$

where $E_{s}$ is the Elastic modulus of the material of the foam and $\rho$ its relative density. As can be observed, and previously commented, these models generally over-predict the values of Young's modulus. This over prediction is more important when compared to the cases of agglomerated foams. 
The experimental foam obtained in the present work by infiltration presented a porosity of $63 \%$ and a Young's modulus of $3.1 \mathrm{GPa}$ (see xE in Fig. 4a, circled), very close to the magnitudes estimated in the present work for the infiltration case (2.8 GPa), and significantly different from the estimation obtained for PM case (1.5 GPa ). Porosity for the case of the experimental foam obtained by PM was $51 \%$, while its Young's modulus reached 1.36 GPa (see xPM in Fig. 4a). For this case Young's modulus used for FEA simulations was $38.2 \mathrm{GPa}$, experimentally determined for a bulk material manufactured by PM (without space holder particles). This led to Young's modulus estimations, for a porosity of $51 \%$, of $2.34 \mathrm{GPa}$ (using the model for infiltation) and $1.52 \mathrm{GPa}$ (using the model for PM). As can be noted, the value estimated using simulations with pores agglomeration (PM) is closer to the experimental value (also circled in Fig. 4a). Fig. 4b presents the relative differences between experimental foams and FEA estimations depending on the agglomeration used in the model (circled). As can be seen, when the correct model is used the relative differences are lower than $10 \%$, increasing to values as high as $110 \%$ for the case of the foam manufactured by infiltration compared to the FEA model using agglomerated pores. This figure also shows (dot line) the relative differences between the Young's moduli calculated by FEA simulations replicating PM (agglomerated pores) and infiltration (non-agglomerated). As can be noted a maximum of $75 \%$ was reached, fact that demonstrates the important effect of the selection of the model and the dependence of the manufacturing process.

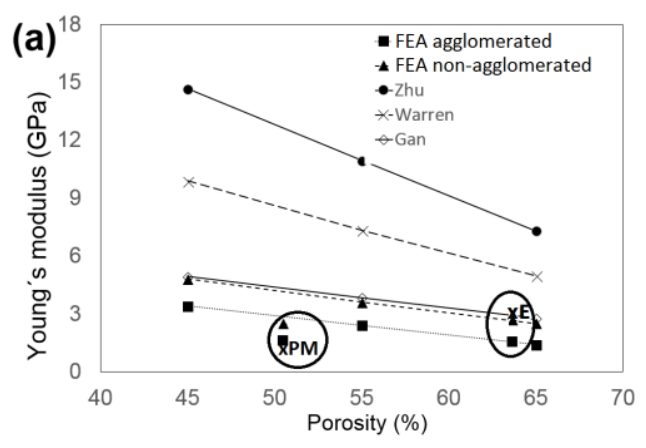

(a)

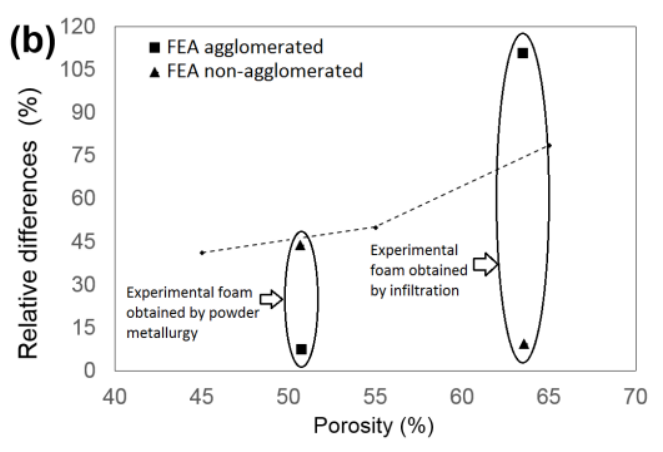

(b)

Fig. 4: (a) Compressive Young's modulus variation for experimental foams and different models depending on the porosity; and (b) relative differences between i) experimental and FEA estimations using models with agglomerated and nonagglomerated pores (circled), and ii) between FEA models (dot line).

Figure 5 shows the mechanical behavior of the experimental foam obtained by infiltration, with the three characteristic regions of the foams: (i) an initial linear elastic 


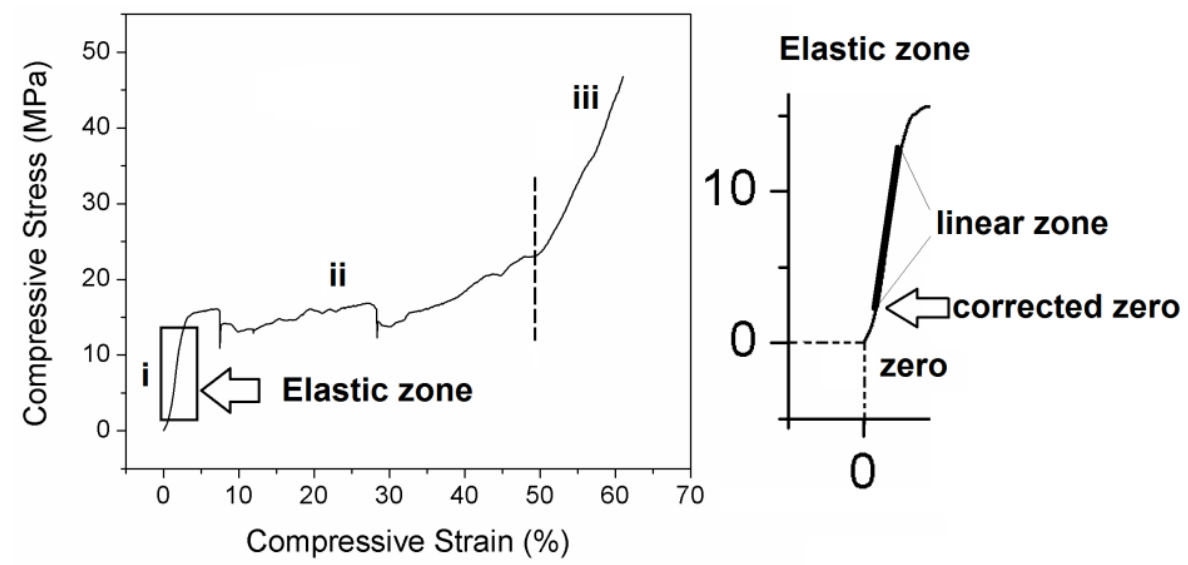

Fig. 5: Compressive stress-strain curve for the experimental foam obtained by infiltration, indicating the correction for the linear zone.

region at very low strain, (ii) an extended plateau region at a relative constant stress level where the stress increases slowly as the cells deform plastically and collapse, and (iii) a densification region where the collapsed cells are compacted together, increasing again the stress [22]. In this figure it is detailed the above mentioned method for Young's calculation correcting the location of zero.

In order to study the effect of agglomeration on the behavior of the foams, an analysis of the Von Mises stresses was conducted, which is presented in Table 1. As can be seen, localized maxima values were obtained for foams with agglomeration, a condition that leads to a decrease in Young's modulus, as already observed in Fig. 4.

Table 1: Maxima Von Mises stresses $\left(\times 10^{5} \mathrm{~Pa}\right)$ for models of foams with different porosities (in \%), recreating foams manufactured by PM and infiltration processes

\begin{tabular}{ccc}
\hline \hline Porosity & PM & Infiltration \\
\hline 45 & $1.58 \pm 0.23$ & $0.85 \pm 0.06$ \\
55 & $1.86 \pm 0.20$ & $1.00 \pm 0.09$ \\
65 & $3.14 \pm 0.26$ & $1.16 \pm 0.08$ \\
\hline \hline
\end{tabular}

It is recognized that an important parameter affecting $E$ is the cell wall thickness $(w)$ [4]. In our case the effect of the agglomeration is also important, as can be observed in Fig. 6a-b, where cross-sections of foams are illustrated with porosities of $55 \%$, without and with pore agglomeration (Fig. 6a and Fig. 6b, respectively). Although agglomeration of pores is an important phenomenon, the study of this problem 
needs further attention. For other materials, e.g particulate composites or nanostructured materials, the investigation regarding the degree of agglomeration goes deeper. Machrafi et al. [23] used the degree of agglomeration of particles $(m)$ in nanocomposites as the ratio between the mean radius of the agglomerates and the size of an individual particle, so for $m=1$ means no-agglomeration. If we observe Fig. 6b, pore clusters can be clearly defined, their sizes being significantly higher than the radius of an individual an isolated pore. In Fig. 6a a pore unit cell (representing the whole material) containing only one pore, while the unit cells in Fig. 6b contain multiple pores. So, $m$ could be re-introduced for porous materials and determined by the ratio between the size of the pore unit cells as follows:

$$
m=s_{a} / s_{p},
$$

where $s_{a}$ and $s_{p}$ are the sizes of the agglomerated and the individual pores, respectively. Therefore, in Fig. 6b, a value of $m=3.2$, indicates approximately the complete pores presented in one-unit cell. For validating purposes, Figs. $6 \mathrm{c}$ and $6 \mathrm{~d}$ show experimental foams obtained by infiltration and PM, respectively. As can be clearly
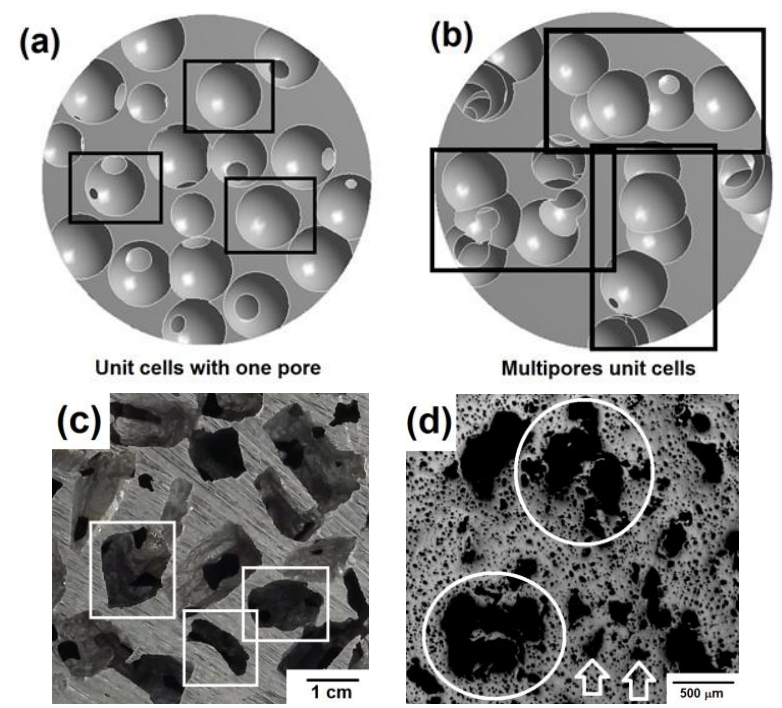

Fig. 6: Representative cross-sections of ANSYS Finite Element Models recreating foams obtained using infiltration (a) and PM (b) processes. Experimental foams oobtained using SHPs by infiltration (c) and PM (d), where unit cells with one pore (squares) and multipores unit cells (circled) are observed. Isolated pores are also observed for PM (arrows). 
observed, DEM-FEA simulations agree with the experimental foams, with high pores agglomeration when PM is used (circled in Fig. 6d).

Table 2 presents the values of $m$ and $w$, including a normalized cell wall thickness $\left(w_{n}\right)$ calculated by dividing $w$ by $m$. For the case of foams obtained using infiltration $m=1$. As can be observed, $w_{n}$ for PM foams are lower than $w$ for infiltration. These results allow for the correlation of Young's modulus: first, $E \sim 1 / m$; and then $E \sim w_{n}$. In fact, $w_{n}$ for PM foams are lower than $w$ for infiltration foams, which could explain the lower values of $E$ for PM foams. Related to the experimental foam, used for validating purposes, it presented an average wall thickness of $2.0 \mathrm{~mm}$, very close to the obtained in the case of infiltration $(2.3 \mathrm{~mm})$, and significantly different of the estimation obtained for PM case $(6.22 \mathrm{~mm})$. This result remarks the important effect of the selection of the model.

Table 2: Values of cell wall thickness ( $w$, in $\mathrm{mm}$ ) for PM and infiltration foams; and degree of agglomeration $(m)$ and normalized cell wall thickness $\left(w_{n}\right.$, in $\left.\mathrm{mm}\right)$ for PM foams

\begin{tabular}{ccccc}
\hline \hline \multirow{2}{*}{ Porosity } & $w$ & $w$ & $m$ & $w_{n}(w / m)$ \\
& PM & Infiltration & PM & PM \\
\hline 45 & $7.82 \pm 0.63$ & $4.31 \pm 0.21$ & $2.4 \pm 0.18$ & $3.26 \pm 0.26$ \\
55 & $7.20 \pm 0.33$ & $3.03 \pm 0.32$ & $3.2 \pm 0.29$ & $2.25 \pm 0.30$ \\
65 & $6.22 \pm 0.58$ & $2.30 \pm 0.25$ & $4.3 \pm 0.25$ & $1.45 \pm 0.12$ \\
\hline \hline
\end{tabular}

\section{CONCLUSIONS}

After FEA analysis of the compressive response of foams modeled with different agglomerations generated by DEM, the following conclusions can be drawn:

1. It was possible to generate models of foams with different agglomeration levels depending on the manufacturing process, using DEM followed by FEA.

2. Young's modulus significantly decreases when the porosity increases for all the modeled foams.

3. For the same porosity, the Young's moduli for foams without agglomeration were significantly higher than for foams with pore agglomeration. The experimental foam obtained by infiltration presented a Young's modulus very close to the model that simulates this process, being significantly far from the PM model, demonstrating the important effect of not only the porosity percentage but also its distribution. 
4. The effect of the cell wall thickness is not the only parameter to be taken into account, but also the degree of agglomeration when predicting Young's modulus. The introduction of a normalized cell wall thickness according to the degree of agglomeration permitted an explanation of the mechanical behavior of the foams.

\section{ACKNOWLEDGEMENTS}

I. Alfonso would like to acknowledge the financial support from SEP CONACYT 285215 and UNAM PAPIIT IN117316. Robin Drew acknowledges the financial support from PREI DGAPA UNAM. L. Pérez acknowledges the financial support from the Advanced Center for Electrical and Electronic Engineering, AC3E, Basal Project FB0008, CONICYT.

\section{REFERENCES}

[1] M.F. Ashby, A.G. Evans, N.A. Fleck, L.J. Gibson, J.W. Hutchinson, H.N.G. WADLEY (2000) "Metal Foams: a Design Guide", Butterworth-Heinemann, Boston.

[2] J. Banhart (2001) Manufacture, Characterization and Application of Cellular Metals and Metal Foams. Materials Science 46 559-632.

[3] A.A. Mohammadi Nasrabadi, R. Hedayati, M. Sadighi (2016) Numerical and Experimental Study of the Mechanical Response of Aluminum Foams under Compressive Loading using Ct Data. (Polish) JTAM 54 1357-1368.

[4] R. Hedayati, M. Sadighi (2018) Low-Velocity Impact Behaviour of Open-Cell Foams. (Polish) JTAM 56 939-949.

[5] J. Xiao, Y. Yang, G. QiU, Y. Liao, H. Cui, X. L (2015) Volume Change of Macropores of Titanium Foams During Sintering. Transactions of Nonferrous Metals Society of China 25 3834-3839.

[6] E.M. Castrodeza, C. Mapelli, M. Vedani, S. Arnaboldi, P. Bassani, A. TUISSI (2009) Processing of Shape Memory CuZnAl Open-Cell Foam by Molten Metal Infiltration. Journal of Materials Engineering and Performance 18 484-489.

[7] N. Jha, D.P. Mondal, J. Dutta Majumdar, A. Badkul, A.K. Jha, A.K. Khare (2013) Highly Porous Open Cell Ti-Foam Using $\mathrm{NaCl}$ as Temporary Space Holder Through Powder Metallurgy Route. Materials and Design 47 810-819.

[8] F. Mat Noor, M.I.M. Zain, K.R. Jamaludin, R. Hussin, Z. Kamdi, A. Ismail, S. Ahmad, H. TAIB (2014) Potassium Bromide as Space Holder for Titanium Foam Preparation. Applyed Mechanics and Materials 465-466 922-926.

[9] L. Pérez, S. Lascano, C. Aguilar, D. Domancic, I. Alfonso (2015) Simplified Fractal FEA Model for the Estimation of the Young's Modulus of Ti Foams Obtained by Powder Metallurgy. Materials and Design 83 276-283. 
[10] J.O. Osorio-Hernández, M.A. Suarez, R. Goodall, G.A. LaraRodriguez, I. Alfonso, I.A. FigueroA (2014) Manufacturing of Open-Cell Mg Foams by Replication Process and Mechanical Properties. Materials and Design 64 136-141.

[11] B.H.G. Jigh, M.A. FARsi, H.H. ToudeshKy (2018) Prediction of the Stress-Strain Behavior of Open-Cell Aluminum Foam under Compressive Loading and the Effects of Various RVE Boundary Conditions. Journal of Materials Engineering and Performance 27 2576-2585.

[12] M. Kirca, A. Gul, E. Ekinci, F. YArdim, A. Mugan (2007) Computational Modeling of Micro-Cellular Carbon Foams. Finite Elements in Analysis and Design 4445 52.

[13] F.V. Antunes, J.A.M. Ferreira, C. CAPEla (2001) Numerical Modelling of the Young's Modulus of Syntactic Foams. Finite Elements in Analysis and Design 47 78-84.

[14] H.X. ZhU, J.F. KNOTt, N.J. Mills (1997) Analysis of the Elastic Properties of OpenCell Foams with Tetrakaidecahedral Cells. Journal of the Mechanics and Physics of Solids 45 319-343.

[15] W.E. Warren, A.M. KrayniK (1988) The Linear Elastic Properties of Open-Cell Foams. Journal of Applied Mechanics 55 341-346.

[16] Y.X. GAN, C. ChEN, Y.P. SHEN (2005) Three-Dimensional Modeling of the Mechanical Property of Linearly Elastic Open Cell Foams. International Journal of Solids and Structures 42 6628-6642.

[17] A. HAS AN (2010) An Improved Model for FE Modeling and Simulation of Closed Cell Al-Alloy Foams. Advances in Materials Science and Engineering 2010 1-12.

[18] J.H. Cadena, I. Alfonso, J.H. Ramírez, V. Rodríguez-Iglesias, I.A. FigueroA, C. Aguilar (2014) Improvement of FEA Estimations for Compression Behavior of Mg Foams Based on Experimental Observations. Computational Materials Science 91 359-363.

[19] L. Pérez, S. Lascano, C. Aguilar, D. Estañ, U. Messner, I.A. Figueroa, I. ALFONSO (2015) DEM-FEA Estimation of Pores Arrangement Effect on the Compressive Young's Modulus for Mg Foams. Computational Materials Science 110 281-286.

[20] P.A. Cundall, O.D. StRack (1979) A Discrete Numerical Model for Granular Assemblies. Geotechnique 29 47-65.

[21] U.A. Joshi, P. Joshi, S.P. Harsha, S.C. Sharma (2010) Evaluation of the Mechanical Properties of Carbon Nanotube Based Composites by Finite Element Analysis. International Journal of Engineering Science 2 1098-1107.

[22] R. Florek, F. SimanČí́K, M. Nosko, J. HARnúŠKová (2010) Compression Test Evaluation Method for Aluminium Foam Parts of Different Alloys and Densities. Powder Metallurgy Progress 10 207-212.

[23] H. Machrafi, G. Lebon, C.S. Iorio (2016) Effect of Volume-Fraction Dependent Agglomeration of Nanoparticles on the Thermal Conductivity of Nanocomposites: Applications to Epoxy Resins, Filled by $\mathrm{SiO} 2$, AlN and $\mathrm{MgO}$ Nanoparticles. Composites Science and Technology 130 78-87. 